\title{
Cultura ecológica de jóvenes universitarios
}

\author{
Evelyng Astudillo Sánchez, Nicole Melendres Vélez, \\ Brunny Espinoza Amén, Marco Moscoso Chávez, Teresa Baquerizo Cornejo
}

\section{Resumen}

El objetivo del presente estudio es conocer el tipo de visión ecológica inherente de los jóvenes universitarios de la Universidad de Especialidades Espíritu Santo (UEES) mediante una encuesta para fomentar actividades que promuevan una cultura ecológica. Por lo tanto, se realizó una investigación de carácter exploratorio - descriptivo con un diseño transversal y un enfoque cuantitativo-cualitativo. La muestra seleccionada está constituida por 350 estudiantes de pregrado, elegidos de forma aleatoria, con un rango de edad entre 18 a 29 años. Se aplicó una encuesta de siete preguntas para conocer la percepción sobre el medio ambiente, la inteligencia ecológica, la conducta resiliente que le permita compensar los daños que pudieran ocasionar sus hábitos. Los resultados indican que los estudiantes (75\%) valoran el medio ambiente; la influencia recibida proviene de la familia $(26 \%)$ y opinión de expertos (26\%); la actividad más común ejercida es el reciclaje $(54.9 \%)$; son conscientes (42.3\%) de que probablemente su conducta contamina el ambiente; y están dispuestos (80.6\%) en tomar acciones ecológicas. Los datos sugieren que los estudiantes han desarrollado una adaptación cultural antropocéntrica y se infiere que todavía no existe una cultura ecológica implantada propiamente dicha, por ello se necesita fomentar hábitos resilientes hacia un desarrollo sostenible. Guiar acciones en el ambiente académico que promuevan una conducta que evidencie una cultura sustentable es imperante.

\section{Palabras clave:}

Percepción ambiental, cultura ecológica, resiliencia ecológica, inteligencia ecológica, desarrollo sostenible.

\section{Abstract}

For the present study, research was carried out of an explorative nature. It was descriptive with a transversal design and a quantitative focus. The selected sample is constituted by 350 undergraduate students, selected at random, between the ages of 18 and 29 at the Universidad de Especialidades Espíritu Santo (UEES). A seven-question questionnaire was handed out to discover their perception about their environment, their ecological intelligence, and any resilient behavior that they might have that would permit them to compensate for any harm that they may cause to their environment. The results of the study indicate that students $(75 \%)$ value the environment; their received influence comes from their family $(26 \%)$ and from expert opinions $(26 \%)$; the activity most utilized by them was recycling $(54.9 \%)$; they are aware $(42.3 \%)$ that their conduct most likely pollutes their environment; and they are prepared $(80.6 \%)$ to take ecological measures. The data suggest that students have developed an anthropomorphic cultural adaptation and it may be inferred that, strictly speaking, there still does not exist an ecological culture instilled. That is why it is imperative to further resilient habits towards sustainable development. To guide our actions in an academic environment that promotes a type of behavior that evinces our responsibilities and commitment towards sustainable development demands our utmost attention.

\section{Keywords:}

Environmental perception, ecological culture, ecological resilience, ecological intelligence, sustainable Development. 


\section{Introducción}

La cultura, desde una acepción moderna, es definida como el "conjunto de valores, de creencias, de símbolos, de técnicas, de modos de pensar que definen a cada sociedad" (Acevedo, 2010, 1) y que siempre se diferencian en relación al grupo social de donde provengan. La Organización de las Naciones Unidas para la Educación, la Ciencia y la Cultura (UNESCO, 1982, 24) menciona que la cultura es el conjunto de rasgos distintivos, sean materiales o espirituales, y que estén o no ligados intelectual o afectivamente a una sociedad.

De manera que, la cultura es inherente al ser humano y este está ligado al ambiente en el que se desarrolla, debido a sus necesidades biológicas que suplir; por lo tanto, interactúa forzadamente con otros organismos modificando su entorno. No obstante, son estos cambios los que están relacionados con la cultura, puesto que "no existe pueblo o individuo inculto, solo existen diversas culturas con variadas formas de desarrollo cultural" (Salazar, 1991, 2); en otras palabras, el ser humano exterioriza su cultura según la sociedad y el medio ambiente en el que se desenvuelve y esta forma de cultura va a impactar o dejar una huella en la naturaleza.

Además de la cultura, otro factor que influye en el cuidado al medio ambiente, es la manera en que se percibe o se conoce sobre él. Por lo que, según el ecologista, G. Tyler Miller Jr., existen dos visiones ambientales que pueden adoptar las personas: visión antropocéntrica y la ecocéntrica $(2007,318)$.

Dependiendo de los valores y del énfasis que posee una cultura hacia el medio ambiente será el impacto o cuidado hacia la naturaleza. Por esto, es necesario que la cultura de una sociedad no sea abordada con una visión antropocéntrica sino que se priorice la interrelación del humano con el medio ambiente y las implicaciones de su impacto. El ser humano, al vivir en ecosistemas urbanos que constituyen sistemas heterotróficos depende de la producción primaria que se genera en otro lugar; por lo que necesita de una visión ecológica (Terradas, 2001, 17) e integral que no amenace la diversidad biológica presente en los ecosistemas naturales y Aurbanos.

En el Ecuador, uno de los principales problemas de la pérdida de biodiversidad es la deforestación de sus bosques provocado por la expansión de los ecosistemas urbanos $y$ la frontera agrícola, lo cual es preocupante ya que el país ocupa el primer lugar a nivel mundial en número de especies por unidad de superficie $(0.017 \mathrm{sp} /$ $\mathrm{km}^{2}$ ) (ECOLAP \& MAE, 2007, 5). Adicionalmente, el país es reconocido por su diversidad cultural, deducido por el mestizaje que ha catalizado la pluralidad de expresiones culturales, gastronómicas, folklóricas, idiomáticas (Astudillo, 2010, 20) y en su Constitución lo define como un país multiétnico y pluricultural representado por 17 pueblos indígenas (De la Torre, $2008,7)$. La diversidad cultural ha derivado en oportunidades de integrar una visión holística que promueva un desarrollo sostenible, mientras se incorpora la inteligencia ecológica en individuos ambientalmente responsables.

De manera que desde el año 1992, el Ecuador viene fomentando la conservación de su biodiversidad, debido a que es signatario de importantes convenios ambientales internacionales; como el Convenio de Diversidad Biológica (CDB) que promueve mediante prácticas ambientales sostenibles, una distribución justa y equitativa de los beneficios (Astudillo, 2010, 17). Por lo tanto, el Gobierno Ecuatoriano tiene como meta impulsar los cuatro pilares del desarrollo sostenible: social, económico, ambiental y diversidad cultural; así, lo mencio- 
na María Fernanda Espinosa, Ministra Coordinadora de Patrimonio, cuando se refiere a que "no va a haber cambio en los patrones de producción y consumo, en la profunda huella ecológica si no existe un cambio cultural, un cambio de valores" (El Tiempo, 2015, 1).

Sin embargo, para que exista este cambio cultural se debe considerar que la cultura es una herencia social transmitida, aprendida comprendida y modificada que emerge en instituciones sociales como la familia, así como en instituciones educativas (Salazar, 1991, 4) y este cambio cultural es imperante en el Ecuador para llegar hacia un desarrollo sostenible; por lo que los centros educativos juegan un rol importante. Por esta razón, el presente estudio surgió de la necesidad de fomentar en jóvenes, una cultura ecológica que incorpore acciones pro ambientales de una manera holística a las diferentes disciplinas en las que se desenvuelven. Consecuentemente, el objetivo fue determinar el tipo de visión ecológica de los jóvenes universitarios de la Universidad de Especialidades Espíritu Santo (UEES), mediante una encuesta para fomentar actividades interdisciplinarias que promuevan una cultura ecológica.

\section{Marco Teórico}

Teoría de la Evolución por Selección Natural

Según Charles Darwin en su obra "El Origen de las Especies" (1879), los individuos mejor dotados son los que mejor se adaptan al medio, sobreviven y transmiten sus características; esto se profundiza en su teoría de la selección natural (Marchisio, Devesa, Rosso, \& Sica, 2012, 26), de manera que los organismos se adaptan al medio de forma que los cambios se mantienen en sus genes según su capacidad de supervivencia. Sin embargo, el ser humano dejó de precisar cambios fenotípicos o evolutivos para adecuarse al medio en el que vive, puesto que su inserción en la cultura le permite modificar su propio entorno. De allí la contradicción que representa el ser humano para la teoría de la evolución por selección natural; ya que es el único organismo que no se adapta al medio sino que lo modifica según sus necesidades. Además de las necesidades biológicas, el ser humano es gregario por naturaleza, lo que implica que viva en sociedad y esta genere una cultura condicionada por su medio ambiente así lo afirma el Grupo Edebé "Cuando, gracias a la cultura el ser humano modifica su entorno, de manera que este le sea más favorable $y$ le permita satisfacer sus necesidades y garantizar su supervivencia, decimos que la cultura tiene un valor adaptativo" (2008, 157).

Aunque es posible cuestionar los modos en que el ser humano usa su cultura para adaptarse al entorno que lo rodea, la respuesta recae en la visión mundial ambiental que configurará un punto crucial en esta interrelación. Por lo tanto, la visión ambiental implica procesos cognoscitivos en los sujetos, para que estos puedan adoptar conductas eficientes en lo que al medio ambiente respecta. Estos fenómenos son explicables a partir de los procesos perceptuales que son llevados a cabo por los sujetos y se entiende por percepción "al proceso por el cual los receptores sensoriales y el sistema nervioso reciben y representan la energía de los estímulos que provienen del ambiente" (Myers, 2011, 231). Dependiendo de cuál sea la interpretación de cada individuo acerca del entorno circuncidante y de sus patrones significativos, entendidos como la asociación de experiencias pasadas con la nueva información receptada, se puede determinar cuál es su sistema de creencias y modo de pensar al tratar temas ecológicos. En otras palabras, se puede deducir que una percepción no es igual en todos los individuos ya que los seres humanos se desarrollan en diferentes 
áreas y pasan por diferentes situaciones y por ende sus conocimientos acerca del mundo que los rodea, siempre serán distintos, únicos, incluyendo también su visión ambiental.

El ecologista, G. Tyler Miller Jr., se ha dedicado a estudiar temas del medio ambiente y el desarrollo sostenible, $y$ ha dividido en dos vertientes: la visión ambiental que pueden adoptar las personas: visiones ambientales antropocéntricas o centradas en las personas y las ecocéntricas o centradas en la vida y el planeta $(2007,318)$.

Los sujetos, cegados en las múltiples actividades del día a día y el consumismo egocéntrico no concientizan la destrucción que hacen al explotar la naturaleza, a esto se le denomina visión ambiental centrada en las personas o antropocéntrica ya que se toma a los seres humanos como el centro del planeta, mientras que las otras especies solo tienen un valor instrumental basados en cuán útiles les resultan (Miller, 2007, 318). Un ejemplo de ello es la deforestación de los bosques tropicales a consecuencia de la explotación maderera, la cual es utilizada como recurso para la obtención de dinero o materiales; asimismo, los automóviles, que usados como medios de transporte son los más grandes contaminantes del aire por el $\mathrm{CO}_{2}$ que emanan disminuyendo la calidad de vida de los habitantes, humanos o no, y los efectos hacia el cambio climático.

La segunda visión propuesta por el autor plantea una opción, en la que los individuos y el ecosistema que los rodea se encuentran a un mismo nivel de condiciones y forman un todo integrado; a esta se le denomina una visión ecocéntrica de la relación (Palavecinos, Amérigo, \& Muñoz, 2010 , 4). Esta visión se adopta debido al planteamiento de que existe una responsabilidad ética de evitar la extinción prematura de especies (Miller, 2007, 319) y el respeto a la biodiversidad estaría por sobre cualquier deseo egoísta, económico y sobre la satisfacción de necesidades superfluas. No obstante, se ha reaccionado tarde ante el deterioro progresivo del planeta y se ha tratado de erradicarlo o mantenerlo en grados leves, pero estas medidas no surgirán efecto a menos que todos se comprometan a aportar en ellas.

\section{Adaptación cultural}

La adopción de una visión antropocéntrica generaría un cuestionamiento sobre las estrategias y técnicas desempeñadas por el ser humano para manipular su entorno; es decir permitiría el deterioro del ecosistema sin considerar las consecuencias de ello. Por otra parte, la visión ecocéntrica conllevaría a un manejo responsable de los recursos y a la búsqueda de un balance, entre el medio y las necesidades de un grupo. Esto hace que el análisis de las actividades de los grupos humanos gire en torno a la identificación de conductas que permitan la adaptación de los grupos a las condiciones del medio que los rodea y que potencialice sus oportunidades de sobrevivencia y desarrollo en un sitio determinado (Valls, 2008, 11).

Los recursos que permiten la adaptabilidad de un grupo humano a un espacio geográfico determinado se identifican en los elementos presentes en su cultura; como la resiliencia e inteligencia ecológica. Pero, al mencionar la cultura humana no se hace referencia a una en particular, sino a la gran y amplia gama de culturas que existen alrededor del mundo. Cada una de ellas tiene una influencia en el entorno en el que se despliegan, debido a que el bienestar humano y el entorno natural tienen una relación intrínseca que se establece a través de los servicios ambientales.

Además, todas las personas, independientemente del estrato social, etnia o raza 
a la que pertenezcan, dependen del capital natural para sobrevivir (Programa de las Naciones Unidas para el Medio Ambiente, $2007,15)$; caso contrario, se consumirían los recursos naturales que son limitados y se degradaría la calidad de vida en el planeta ya sea en su diversidad biológica y cultural.

El escritor libanés, Amin Maaluf expresa en su ensayo Identidades Asesinas (2009, 76), lo siguiente: “¿Por qué habríamos de preocuparnos menos por la diversidad de culturas humanas que por la diversidad de especies animales o vegetales? Ese deseo nuestro, tan legítimo, de conservar el entorno natural, ¿no deberíamos extenderlo también al entorno humano?" Este autor, en su ensayo, manifiesta cómo la humanidad se aniquila entre sí debido a conflictos por las diferencias étnicas y culturales, pero también se observa una connotación que no debe malinterpretarse con el abandono del cuidado al medio ambiente, sino que resalta lo relevante de la diversidad cultural y su interacción con los recursos naturales.

Lo que se pretende manifestar es que para que exista una conservación de la naturaleza, se debe recurrir a un cambio en los diferentes sistemas culturales y la influencia que estos ejercen en su hábitat o entorno de residencia. El cambio enfrenta a dos visiones diferentes del mundo: el egocentrismo con énfasis en la capacidad de la ciencia y la tecnología para solventar la actual problemática ambiental, frente a la vanguardia empeñada en un cambio radical hacia una sociedad sostenible y amigable con el ambiente (Palavecinos, Amérigo, \& Muñoz, 2010, 4).

\section{La cultura ecológica}

La adaptabilidad cultural del ser humano supone una influencia en el medio ambiente en el que se desenvuelve debido al nivel de consumo del capital natural, pero este grado de influencia dependerá de los componentes culturales implicados en la visión ambiental adoptada previamente. Por ejemplo, la huella ecológica es un indicador de la sostenibilidad que mide el impacto ambiental producido por el nivel de consumo antrópico y la capacidad de la tierra para regenerar sus recursos, donde el nivel de consumo está asociado a la cultura y varía entre naciones.

Así lo evidencia el Informe Planeta Vivo 2014 (13) al mencionar que:

"Si todas las personas del Planeta tuviesen la Huella promedio de un residente de Qatar, necesitaríamos 4,8 planetas. Si tuviéramos el estilo de vida de un residente típico de los Estados Unidos, necesitaríamos 3,9 planetas. La cifra para un residente típico de Eslovaquia o Corea del Sur sería 2 o 2,5 planetas, respectivamente, mientras que un residente típico de Sudáfrica o Argentina necesitaría 1,4 o 1,5 planetas, respectivamente".

Entonces, resulta necesario recordar que la cultura es un conjunto de conocimientos aprendidos dentro de una comunidad (Mosterín, 2008, 57) pero para tener una cultura ecológica, deben observarse algunos criterios, por lo que $\mathrm{E}$. Leff, en su libro Racionalidad Ambiental: La reapropiación social de la naturaleza $(2009,221)$, haciendo referencia hacia la cultura ecológica menciona:

"Los seres humanos debemos adquirir la cultura ecológica para estructurar y dar paso a la mediación entre la sociedad y el medio ambiente, combinando los diversos estilos étnicos propios de cada individuo y el aprovechamiento racional de los recursos naturales".

En efecto, la cultura ecológica encierra tanto a las pautas culturales y al cuidado del medio ambiente como método aplicable 
para el mantenimiento de la vida, tanto humana como vegetal y animal, siendo el equilibrio entre el desarrollo y progreso de la sociedad conjuntamente con la naturaleza para garantizar la vida y la biodiversidad.

Sin embargo, esta preocupación por conocer el medio ambiente no es algo reciente, ya que ha ido surgiendo a lo largo del tiempo; prueba de esto es que el ser humano ha incursionado en nuevas técnicas y diseñado tecnología que le permita preservar su entorno. Esto ha generado que, a nivel académico, surja una sub-rama derivada de la ecología que explique los diversos cambios culturales basados en una reconsideración y apreciación del medio ambiente. La etnocología es la rama de la ecología que se interesa en las relaciones entre las diversas culturas y la diversidad biológica, a más de concentrarse en ahondar sobre los impactos y la interrelación del hombre con su medio ambiente (Reyes \& Martí, 2007, 1). Esta sub división de la ciencia se puede considerar como un punto de abordaje hacia la cultura ecológica, puesto que busca vincular a los diferentes grupos humanos con su hábitat $y$ permite la reflexión del impacto que han producido en él.

Por lo tanto, la cultura ecológica no solo es una apreciación de los elementos culturales; sino que responde a un proceso de investigación sistematizada que invita a generar discusión sobre las diversas situaciones ambientales que surgen del pensamiento colectivo de un grupo humano en un lugar determinado. En otras palabras, los diversos impactos generados por una sociedad que se desenvuelve en un medio ambiente particular afectarán ciertos recursos naturales que serán temas de discusión en la comunidad científica; por ejemplo, los recursos pesqueros como el pescado, camarones que han sido sobreexplotados por el consumo masivo por países como China o
India (FAO, 2002, 3).

\section{Resiliencia Ecológica}

Las culturas no son organismos estáticos. Conforme pasa el tiempo, es necesario cambiar y llevar a cabo procesos adaptativos frente a los nuevos retos que se suscitan en los ambientes sociales. Muchos de estos cambios no suelen ser positivos y obligan a poner en marcha los recursos tanto materiales como cognitivos y cooperativos con los que cuenta una sociedad. La forma en que esos recursos son usados en momentos difíciles responde al nombre de resiliencia; la cual es concebida por Grotberg como "la capacidad del ser humano para hacer frente a las adversidades de la vida, aprender de ellas, superarlas e inclusive ser transformados por ellas" $(2006,18)$. Otros autores también la definen como la capacidad de adaptación bajo fuentes generadoras de tensión (Papalia, Wendkos, \& Duskin, 2010, 674).

Esta forma de superar las adversidades sirve como modelo para analizar las formas en que el medio ambiente también ha logrado regenerarse $y$ ser productivo después de eventos perjudiciales tales como incendios, sequias extensas, falta de nutrientes, impactos antrópicos, entre otros. Sánchez $(2011,248)$ señala que esa capacidad de regeneración de la tierra y autocuración puede ser entendida como la resiliencia ecológica dada por "la cantidad de cambio que un sistema puede soportar, e incluso así, aún poder seguir adaptándose a las condiciones cambiantes".

Se proporciona un ejemplo que muestra la resiliencia socio - ecológica en la población de Agua Blanca, ubicada en el interior de Parque Nacional Machalilla (PNM), Manabí - Ecuador quienes han cambiado sus actividades insostenibles como la caza, ganadería, tala de madera y producción de carbón vegetal por actividades sostenibles como el turismo ecológico 
y cultural. El ecosistema del PNM ha sido impactado con las prácticas insostenibles que mantenían las poblaciones locales y en el transcurso de 10 años se puede observar la recuperación de la cubierta vegetal, la presencia de aves y mamíferos. La población ha centrado sus actividades en el turismo comunitario como una alternativa económica que genera ingresos y son repartidos equitativamente en la población. Por lo tanto, la relación naturaleza - cultura muestra su condición resiliente de auto organización con el objetivo de lidiar con el cambio a niveles sostenibles (Escalera \& Ruiz, 2011, 120).

La importancia de la resiliencia dentro de la cultura ecológica se fundamenta en el hecho de que el ser humano al ser consciente de la capacidad de autoregeneración de la Tierra y del mantenimiento de sus procesos naturales, procurará no alterarlos con sus actividades diarias para que estos puedan seguir su curso natural.

Por otro lado, entre los factores que pueden influir en la resiliencia de un ecosistema se destacan los mencionados por el consultor ambiental, Marcelo Hernández $(2009,4)$ en su artículo La Resiliencia, clave del desarrollo sostenible. Él menciona que para determinar la existencia o no de resiliencia en un entorno, es necesario analizar:

- Si la biología y ecología son inherentes de sus especies componentes o hábitats.

- La condición de estos componentes individuales previo a un evento.

- La naturaleza, severidad y duración de los impactos suscitados en un medio ambiente determinado.

- Si el grado del impacto potencial ha sido reducido o eliminado.

Estos factores son muy influyentes al tratarse de la resiliencia de un ecosistema y deben ser considerados como indicadores de cuán resiliente es un hábitat para su conservación y mantenimiento. Si se adhie- ren a los indicadores, la vida que alberga en él no se extinguiría y podría continuar con sus procesos naturales.

\section{Inteligencia ecológica}

Parte del proceso de reconocer y poner en práctica las capacidades de un grupo humano para salir de un problema o situación difícil; requiere que se ejecuten acciones organizadas y planificadas para alcanzar metas que produzcan bienestar a nivel general. Dado que la inteligencia es la capacidad para resolver problemas (Myers, 2011,180 ), cuando se hace referencia a situaciones donde se involucra el medio ambiente y este es el afectado por alguna situación; la forma en que se resuelve dicha situación es denominada "inteligencia ecológica".

Goleman $(2009,62)$ estudia las interacciones de las personas con el medio ambiente y la forma en que ellas usan los recursos del mismo, y dirá que la inteligencia responde a "la capacidad de emplear nuestro conocimiento de las consecuencias de la acción humana para hacer el mínimo deterioro a los ecosistemas". Por lo tanto, se involucra al ser humano a las consecuencias de sus acciones y a tomar responsabilidad de aquello que ejecuta y que afecta de alguna manera al medio ambiente.

La aplicación de la inteligencia ecológica, permitiría que los procesos y medidas que prevengan el deterioro ambiental, sean mucho más eficientes por la creación de una autoconciencia que anticipe los efectos ecológicos de nuestras acciones. Según Harper \& Row Inc $(2009,37)$ la autoconciencia es debido al aprendizaje que "permite a los organismos adaptarse y obtener la ventaja más efectiva que proporciona una variedad de oportunidades más amplia". Esta autoconciencia dará como resultado una adquisición de la cual se debe evidenciar una cosmovisión y modo de vida basado en el desarrollo sostenible y el cuida- 
do al planeta.

Desafortunadamente, no todos los seres humanos reconocen la importancia de la resiliencia e inteligencia ecológica debido a que así como existe una diversidad de culturas, existe una diversidad de visiones ambientales donde están construidas las percepciones de cada ser humano sobre el estado de su entorno. Por ejemplo, la nacionalidad Huaorani que habita en la región amazónica del Ecuador tiene su propia cosmovisión de la relación hombre - naturaleza y el propio estado lo reconoce al declarar su territorio como un área protegida, conocida como el Parque Nacional Yasuní. Por otra parte, la cosmovisión occidental que ve a la naturaleza como un capital económico, debido a la explotación petrolera, ha invadido el territorio Huaorani y ellos han adaptado esta visión capitalista que no involucra una cultura ecológica sostenible. No obstante, existe un grupo de ellos, los Tagaeri, quienes han preferido el no contacto con la civilización y viven aisladamente de forma sostenible con el medio ambiente; es decir, mantienen actividades como la caza, pesca y recolección de frutos.

\section{Desarrollo Sostenible}

El cambio climático, derivado del calentamiento global, es el mayor problema ambiental que la sociedad mundial actual enfrenta, ya que provoca el deshielo de glaciales por el aumento de las temperaturas, el cual tendría secuelas en la economía (e.g. transporte, agricultura, seguros y defensa de inundaciones), en la salud (e.g. aumento de temperaturas extremas, expansión de enfermedades y efectos en la población infantil más vulnerable) y efectos sociales (e.g. migración).

Debido a los problemas ambientales que deterioran la calidad de vida de los organismos vivos se hace imperiosa la necesidad de una cultura ecológica y sus nociones implícitas (resiliencia e inteligencia ecológi- cas) para un cambio a nivel de cognición y de comportamiento. Por lo tanto, el desarrollo sostenible es un cambio cognitivo que permite administrar las relaciones de los humanos con su entorno físico y social sin malgastar los recursos naturales y que puedan ser conservados para generaciones futuras (Novo, 2006, 362).

Además, el desarrollo sostenible descrito como un "desarrollo que satisface las necesidades de la generación presente, sin comprometer la capacidad de las generaciones futuras de satisfacer sus propias necesidades (Asamblea General de las Naciones Unidas, 1987, 1) es un paradigma global de las Naciones Unidas que fue concebido ante la urgencia de reducir y mitigar los problemas ambientales crecientes. El paradigma de desarrollo sostenible se basa en cuatro pilares o dimensiones: la sociedad (social), el medio ambiente (ecológico), la economía (económico) y la cultura (diversidad cultural, incluida desde el 2001).

Para la ejecución de este, se debe impulsar las economías locales fortaleciendo su capacidad de gestión e implementando políticas públicas ambientales. Por ende, el desarrollo sostenible comprende un compromiso de transformación radical en el estilo de vida humana y plantea la conservación de los recursos naturales de una forma más eficiente y mediante una distribución justa. Esto implica, además, un consumo responsable y la explotación controlada de los recursos del planeta; así lo menciona Simental $(2010,93)$ cuando dice que "El ser humano construye su tecnósfera dentro del espacio que le arrebata a la biósfera, limitándola, cercándola, privándole espacios que son, a final de cuentas, vitales para el ser humano".

El cambio hacia una cultura ecológica Hay que tener en cuenta que el desarrollo sostenible implica una conversión no sólo 
de las acciones o las actividades diarias del ser humano, sino también de la manera en la que se visualiza al medio ambiente; este cambio podría manifestarse a través de una visión ecocéntrica. Es probable que las personas con una visión antropocéntrica, no deseen y se nieguen a visualizar el daño que el ser humano le hace al medio ambiente, pero es un hecho el fenómeno del calentamiento global que es ocasionado por el mal uso de recursos, la sobreexplotación de los bosques, la acumulación de desechos, la sobrepoblación humana, la contaminación atmosférica, la erosión, entre otros (Edinun, 2009, 42).

Las medidas que contrarresten estos problemas ambientales resultan necesarias, pero muchas actividades ecológicas en respuesta quizá no surtan efecto porque son momentáneas; es decir no implican un cambio a nivel cognitivo como sí podría hacerlo la adjudicación de una cultura ecológica.

El desarrollo sostenible y cultura ecológica, son dos términos que van relacionados por la vinculación de un tercer término denominado conducta sustentable, que involucra las acciones efectivas y deliberadas del individuo a favor de la preservación de los recursos naturales para el bienestar individual y social de las generaciones presentas y las que vendrán (Corral-Verdugo \& Pinheiro, 2004, 11). Para la implementación de una cultura ecológica se necesita de un plan de acción a través de la educación orientado hacia la preservación y acercamiento de los individuos a los diversos entornos naturales. Por ello, la UNESCO ha implementado su programa de Educación para el Desarrollo Sostenible (EDS) y dentro de este se encuentra la Educación sobre el Cambio Climático (ECC), el cual ayuda a fortalecer las capacidades para entender las consecuencias del cambio climático y amplía el conocimiento en los jóvenes sobre el clima.
Mediante la educación se espera promover una conducta sustentable en cada individuo que vive una cultura ecológica y posee un estilo de vida sustentable, y que en conjunto conforman la posibilidad de cambio que las sociedades deberían implementar en sus planes poblacionales. Un ejemplo claro, de dicho cambio, lo conforman los ciudadanos de la ciudad de $\mathrm{Hy}$ derabad en India cuya población excede los cinco millones de habitantes. Esta ciudad para incrementar la calidad de vida de los residentes ha decidido plantar árboles y parques en áreas donde estuvieron construidas fábricas contaminantes e incluso se han creado "edificios verdes" que consuman menos agua y electricidad (Douglas, 2009, 85). Como se ha evidenciado en el caso de la ciudad de Hyderabad, el cambio que propone este estudio no es imposible e incluso resulta beneficioso para la sociedad misma, ya que el invertir en un modo de vivir amigable con el ambiente, eleva la calidad de vida de los habitantes. Según Cordera $(2009,3)$ una inversión en la conservación del medio ambiente corresponde a una educación de la sociedad y a la investigación. Una sociedad educada a tener una cultura ecológica y a que sus actividades sean sustentables, no solo vivirá mejor sino que pondrá a salvo la diversidad biológica, la cual será el patrimonio a entregar a las futuras generaciones.

Ante esto puede surgir la interrogante sobre dónde y quién es el encargado de brindar o educar a la sociedad y que esta pueda ser sensibilizada en este aspecto. Diferentes autores sugieren que la respuesta yace en los institutos de educación superior, pues es dentro de ellos donde la educación a la sociedad y la investigación son tareas primordiales enfocadas en las necesidades del medio circundante (Vitarelli, 2007, 127). Los jóvenes son los precursores de los cambios sociales, y para que haya un cambio con el fin de reducir el daño ambiental, la cultura ecológica debe 
ser introducida en la adquisición de los valores culturales de aquellos jóvenes que conducirán a un cambio trascendental en la sociedad de la que forman.

\section{Metodología}

La investigación es de carácter exploratorio - descriptivo con un diseño transversal y un enfoque cuantitativo-cualitativo (Hernández, Fernández, \& Baptista, 2010,86 ). Debido a que la población no ha sido estudiada antes, se busca establecer si en la muestra se hallan rasgos de cultura, de inteligencia ecológica, de resiliencia ecológica y la noción sobre el desarrollo sostenible.

La muestra seleccionada está constituida por 350 estudiantes de pregrado, elegidos de forma aleatoria, con un rango de edad entre 18 a 29 años, que estuvieron matriculados en el semestre de verano 2014 y que se encontraron asistiendo regularmente a clases en el campus de la Universidad de Especialidades Espíritu Santo (UEES) ubicado en el km 2.5 vía Samborondón, en la ciudadela Tornero III.

Para recopilar la información deseada se elaboró una encuesta estructurada de siete preguntas que pretendió levantar información sobre la valoración de los individuos hacia el medio ambiente y las fuentes que influencian a los estudiantes a adoptar una conducta ecológicamente amigable.

Además, la encuesta incluyó las actividades que ellos desempeñan para compensar los daños y la valoración que tienen de sus propias actividades en relación a la contaminación que producen, la predisposición a participar de iniciativas ecológicas y el conocimiento que poseen con respecto al desarrollo sustentable.

Cada pregunta está elaborada para contrastar la información presentada en el marco teórico, junto con la realidad de la muestra a analizar:

- Pregunta 1: Permite realizar una aproximación de valoración ambiental antropocéntrica o ecocéntrica.

- Pregunta 2: Permite analizar la fuente de influencia sobre la valoración del medio ambiente y actúa como reforzador de la primera

- Pregunta 3: Permite apreciar las actividades que han influido a la construcción de la valoración y complementa tanto a la pregunta 1 como a la 2

- Pregunta 4: Analiza el grado de consciencia sobre las actividades contaminantes que los sujetos realizan, lo cual es un indicador de inteligencia ecológica.

- Pregunta 5: Permite apreciar la comprensión del rol de los estudiantes y la valoración de que ellos se vinculen a actividades de preservación ambiental; lo cual es un rasgo de inteligencia y resiliencia ecológica.

- Pregunta 6: Evalúa la predisposición hacia ejecutar una acción concreta en beneficio del medio ambiente, lo cual es un rasgo de la presencia de una cultura ecológica

- Pregunta 7: Permite apreciar si el grupo muestra conoce o no lo que es el desarrollo sostenible y complementa todas las anteriores ya que el desarrollo sostenible involucra todos los otros aspectos.

Para el procesamiento de información se empleó un método relacional-descriptivo a través de diagramas de exposición de información para relacionar la información teórica recopilada y los resultados obtenidos de la muestra de estudio. 


\section{Discusión y Análisis de Resultados}

Se procedió a una elaboración de encuestas compuestas de 7 preguntas cada una, dirigidas a los estudiantes de la Universidad de Especialidades Espíritu Santo, cuyos resultados fueron los siguientes:

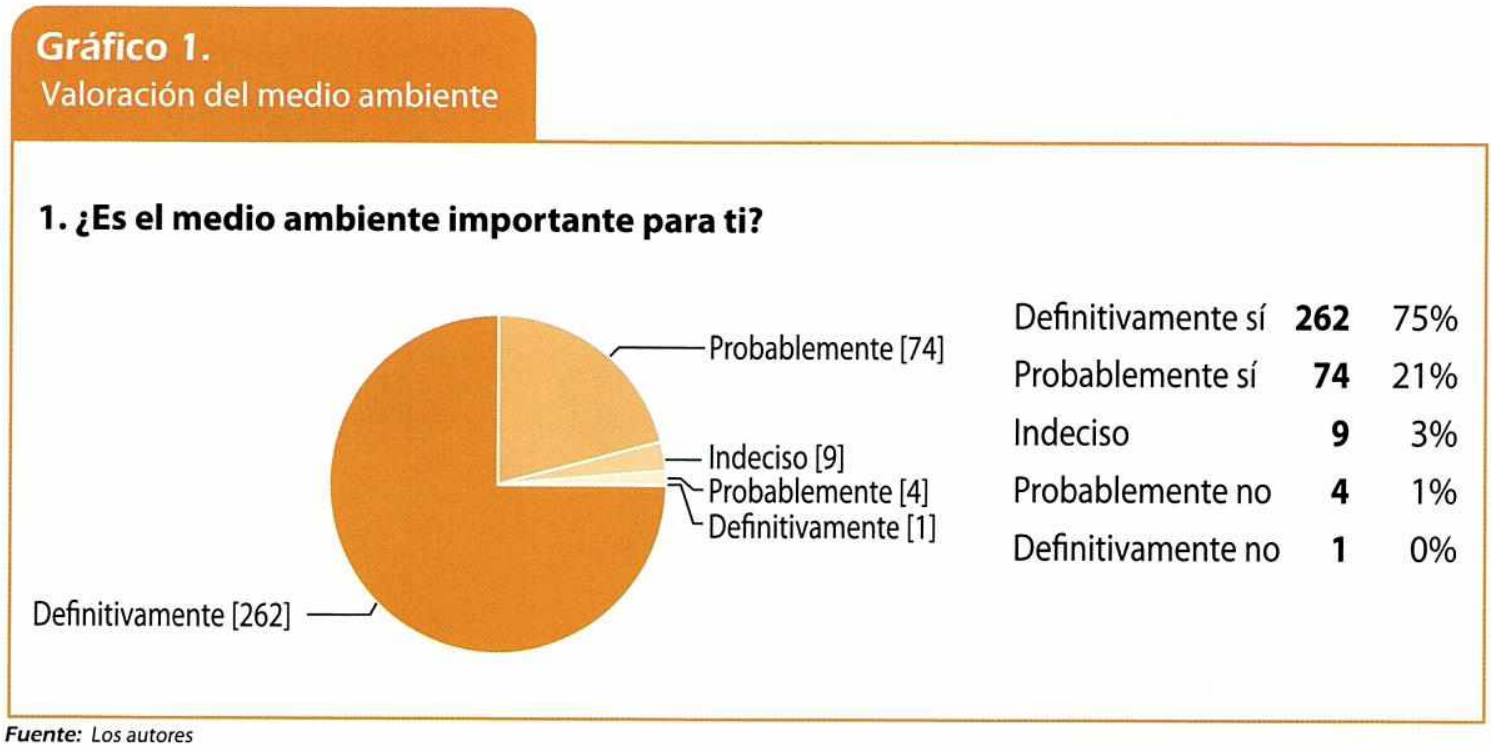

Como se observa en el gráfico 1, existen 262 estudiantes encuestados que manifiestan la gran importancia al medio ambiente siendo aparentemente una cualidad necesaria de una visión ecocéntrica. Por otro lado, el $4 \%$ de los encuestados demuestran tener cierto grado de inseguridad el cual puede sugerir una visión antropocéntrica.

\section{Gráfico 2.}

\section{Origen de la influencia recibida para el cuidado del medio ambiente}

2. Ordene las siguientes opciones con sus respectivos números según la influencia que has recibido acerca del cuidado del medio ambiente

1. Familia • 2. Amigos/Conocidos • 3. Tus profesores • 4. Tu facultad • 5. Opciones de Expertos

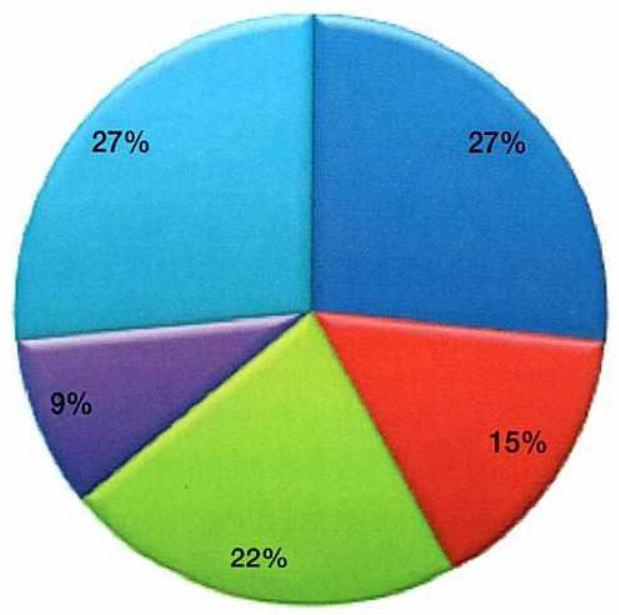

1. Familia

ㄹ. 2. Amigos/Conocidos

$\square 3$ Tus profesores

4. Tu facultad

5. Opciones de

Expertos
$9026 \%$

$5218 \%$

$75 \quad 21 \%$

$329 \%$

$90 \quad 26 \%$ 
En la pregunta 2 se estableció una jerarquía en referencia a la fuente de influencia en torno al cuidado ambiental, por lo que el gráfico presenta la primera opción elegida por los encuestados. En el gráfico 2, se observa que la familia y las opiniones de los expertos influyen en un grado similar, casi al mismo nivel, lo que permite sugerir que el cambio se inicia en el núcleo familiar. La familia es el centro donde los jóvenes adquieren sus patrones de conducta y se hace adjudicadora del papel cultural indicado por el filósofo Mosterín, por la enseñanza de conocimientos hacia los individuos $(2008$, 57). Los conocimientos tam- bién son luego reforzados por las opiniones de los expertos, quienes tienen el poder y la trascendencia en la elección de la visión ambiental escogida por los jóvenes, según la gráfica 2 .

Mientras que en el último lugar se encuentra la Facultad de Artes Liberales, los jóvenes no se sienten incentivados por esta entidad en sí hacia el cuidado ambiental, lo que indica que se debe reforzar esta área porque será de gran relevancia en la formación y difusión de los cambios educacionales en torno al cuidado del medio ambiente.

Gráfico 3.

Formas de influencia en el cuidado del medio ambiente

\section{3. ¿De qué manera han influido en tu percepción para el cuidado del medio ambiente?}

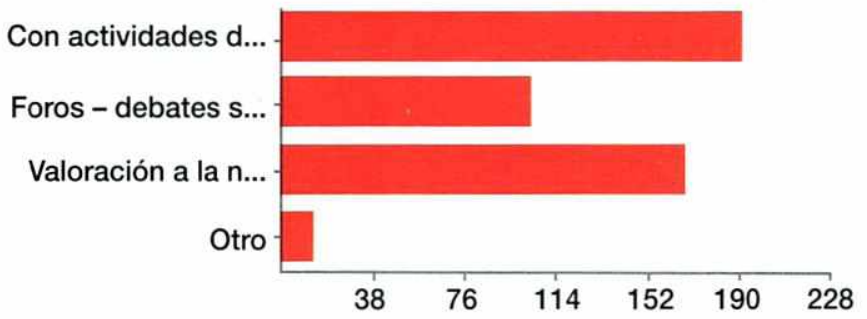

Con actividades de reciclaje, reuso y reutilizar

La pregunta 3 va en relación a la anterior, en la cual los encuestados indicaron quienes son los más influyentes en su percepción ambiental, concibiéndola como el proceso cognitivo representador de los estímulos sensoriales externos (Myers, 2011, 180). Con la diferencia de que aquí especifican la forma en la que recibieron esta información para ser elemento base de su sistema de creencias. Como se observa en el gráfico 3, el 55\% de la muestra concordó en que el reciclaje ha sido el principal y el más común acercamiento que han tenido al cui- dar el medio ambiente. Con un $48 \%$ le sigue la valoración a la naturaleza que simboliza el grado de conciencia ambiental que poseen, debido a que si valoran al medio ambiente, procederán a protegerlo y conservarlo a posterioridad. El 30\% de los encuestados manifestaron que son los foros y debates los que influyen en su perspectiva valorativa; si bien es cierto, son muy útiles a la hora de intercambiar opiniones, conocimientos y perspectivas ya que son procesos que fortalecen la visión ambiental de los individuos. El 14\% indicó que reali- 
zaba otras actividades y entre las respuestas que compartieron sobresalen la presentación de videos y documentales, que pueden también ser muy beneficiosos para adquirir conocimientos del planeta por la cantidad de información que poseen situaciones reales ambientales presentes en otras sociedades o países.

\section{Gráfico 4.}

Reconocimiento de inteligencia ecológica

\section{4. ¿Consideras que tus acividades diarias contaminan al entorno en que te desenvuelves?}

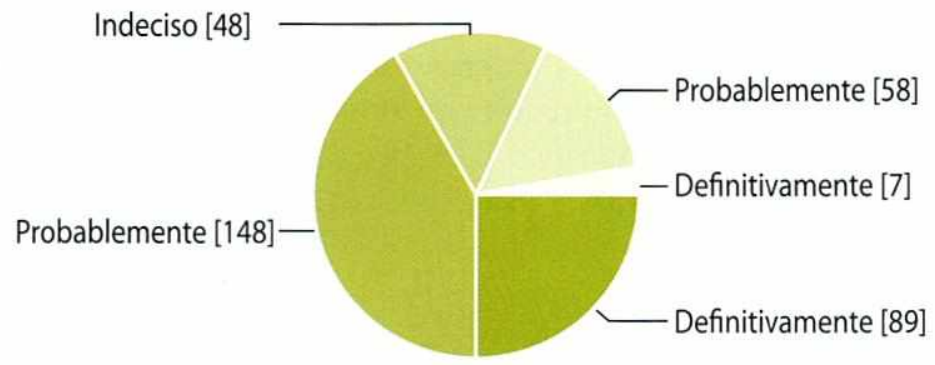

Definitivamente sí

$8925.4 \%$

Probablemente sí

$148 \quad 42.3 \%$

Indeciso

$48 \quad 13.7 \%$

Probablemente no

$5816.6 \%$

Definitivamente no

7

Fuente: Los autores

Por otro lado, la pregunta 4 identifica la autoconciencia ambiental o inteligencia ecológica de cada individuo ya que vincula el reconocimiento de sus propias acciones. Resulta necesario recalcar que es el ser humano el principal responsable de la contaminación al alterar los procesos natu- rales del planeta; no obstante, la muestra estudiada reconoce esa responsabilidad debido a que según el gráfico 4 , un $25,4 \%$ marcó como respuesta un "Definitivamente sî". Mientras que un $16,6 \%$ y un $2 \%$ todavía no tienen ningún indicio de haberla adquirido.

\section{Gráfico 5.}

Nivel de predisposición hacia una cultura ecológica

\section{5. ¿Crees que los jóvenes de hoy deberian tomar acción en actividades ecológicas?}

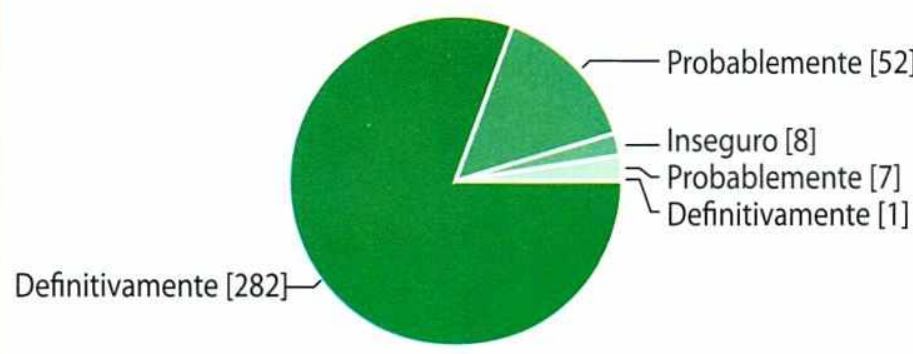

Definitivamente sí $\mathbf{2 8 2} 80.6 \%$

Probablemente sí $\mathbf{5 2} \quad 14.9 \%$

Inseguro $\quad 8 \quad 2.3 \%$

Probablemente no $\quad \mathbf{7} \quad 2 \%$

Definitivamente no $\quad 1 \quad 0.3 \%$ 
La pregunta 5 fue formulada con el propósito de indagar en la capacidad de participación que están dispuestos a ofrecer los jóvenes. En esta pregunta converge una motivación construida en el aprendizaje, la cultura y la interacción social. Según el gráfico 5, un $81 \%$ expresó que definitivamente los jóvenes deben ser parte de actividades ecológicas.

Esta decisión sería un paso a la mediación entre la sociedad y el medio ambiente que dará como resultado la formación de una Cultura Ecológica (Leff, 2009, 221).

\section{Gráfico 6.}

Actividades realizadas para cuidar el medio ambiente

\section{6. ¿Qué actividades para cuidar el medio ambiente realizas?}

Escoje al menos 3 opciones (excepto si escoges "ninguna") y ordenalas en orden de importancia $=1$. Venta de Reciclaje de periódicos . 2. Uso de focos ahorradores en el hogar . 3. Medidas para ahorrar el agua . 4. Reciclaje y/o Venta de botellas de plástico/vidrio . 5. Uso de gasolina ecológica .

6. Elaboración de manualidades con materiales reciclados. 7. No depositar basura en las calles .

8. Ninguna

$$
\begin{aligned}
& \text { - } 1 \text {-Venta de Reciclaje de Periódicos } \\
& \text { - 2. Uso de focos ahorradores en el } \\
& \text { hogar }
\end{aligned}
$$

- 3. Medidas para ahorrar el agua

- 4. Reciclaje de botellas de plástico/vidrio

- 5. Uso de gasolina ecológica

- 6. Elaboración de manualidades con materiales reciclados

- 7. No depositar basura en las calles

8. Ninguna

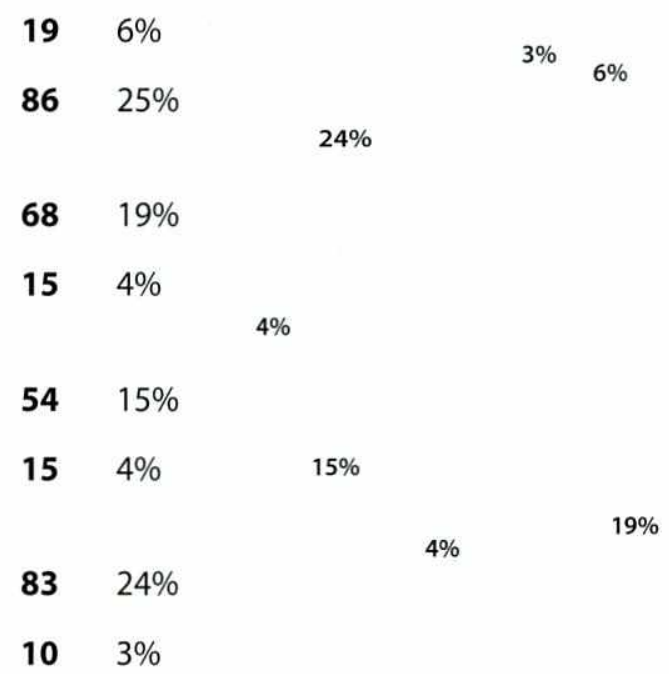

Fuente: Los autores

Al igual que la pregunta No. 2, esta pregunta 6, se enfoca en identificar la jerarquía de las actividades ecológicas que realizan los encuestados. Para mostrar los resultados se toma en cuenta la primera opción que respondieron como la más importante y frecuente para ellos. Véase el siguiente gráfico 6 .

El uso de focos ahorradores y el no depositar basura en las calles son las accio- nes que gozan de mayor popularidad entre los jóvenes sumando el $49 \%$ entre ambos. Le siguen las medidas para ahorrar el agua y el uso de gasolina ecológica, que ayudan respectivamente a no malgastar un recurso vital para la subsistencia y a no contaminar con CO2 la atmósfera. Estas acciones reflejan a nivel conductual la asimilación tanto de la resiliencia como la inteligencia ecológica, factores claves para vivir en armonía con el medio ambiente. 
Gráfico 7.

Comprensión del término Desarrollo Sostenible

\section{De las siguientes opciones elije a tu criterio, cuál se ajusta al término de desarrollo sustentable}

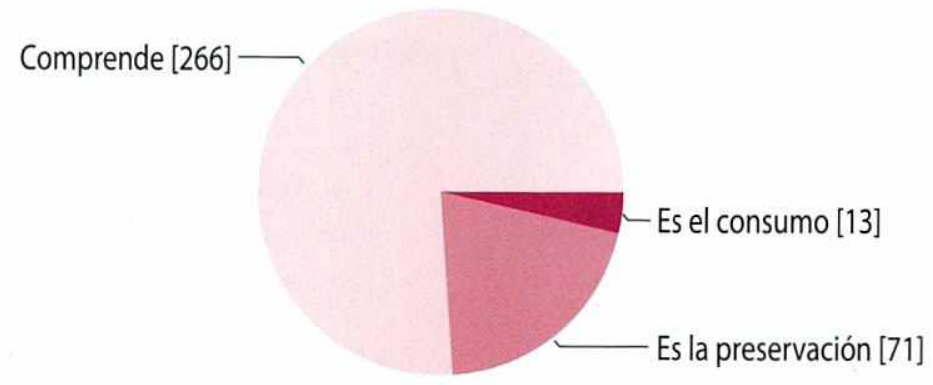

Es el consumo excesivo e irracionales de los recursos naturales

Es la preservación de los recursos naturales a corto plazo para beneficio exclusivo del hombre

La pregunta 7, tuvo la finalidad de evaluar, a manera de prueba, si los estudiantes comprenden el enunciado del término desarrollo sostenible para probar si existe confusión en relación al término y el desarrollo de actividades ambientales. El 76\% acertó en la elección de la definición de desarrollo sustentable por lo que es posible extraer que los sujetos que componen la muestra estudiada conocen del tema y no es ajeno a sus vidas personales.

\section{Conclusiones}

Los datos indican que existe un conocimiento de ciertos conceptos relacionados con el cuidado ambiental, en su mayoría adquiridos por la influencia familiar y de expertos que han recibido la mayoría de los jóvenes. Aunque en menor grado los docentes y la facultad influyen en la captación de ese nuevo conocimiento, ellos serían una herramienta para implementar actividades que desarrollen a sus alumnos una inteligencia ecológica en favor de un desarrollo sostenible. Los estudiantes reconocen que el medio ambiente es importante, lo cual sugiere que su adaptación cultural tiene un enfoque ecocéntrico. Sin embargo, sus otras respuestas generan un contraste al presentar un grado de duda en el reconocimiento de sus actividades antrópicas como un indicador en la disminución de su calidad de vida. Ello indica que todavía no existe una cultura ecológica implantada en los jóvenes universitarios debido a que existe una deficiente inteligencia ecológica. La carencia de la inteligencia ecológica está asociada a la falta de reconocimiento del impacto ambiental y esto sumado a una débil conducta sustentable ligada a las pocas acciones realizadas para compensar o disminuir nuestra huella ecológica.

Sin embargo, el $25 \%$ manifestó que realizarían un cambio sustentable, lo cual es un indicador de efectividad al desarrollo de una resiliencia e inteligencia ecológica que incluya acciones y actividades que no alteren ni perjudiquen al entorno. Aunque intentar adjudicar en los estudiantes una cultura ecológica que implica un estilo de vida sostenible, es un proceso que involucra el medio más influyente como la familia y un proceso formativo en un centro educativo, existe la vigente necesidad de conservar el medio ambiente, lo cual son dos procesos 
(e.g. conservar y educar) que implican tiempo.

\section{Recomendaciones}

El presente estudio presentó algunas limitaciones para su realización y ejecución. La muestra analizada, al responder a un patrón aleatorio de selección, no permite extraer conclusiones particulares que respondan a las realidades contextuales de los sujetos; sino que permiten generar una visión general sobre ciertos factores relacionados a la cultura ambiental, inteligencia ecológica e impacto-beneficio de las acciones que los sujetos llevan a cabo. En un futuro se podría controlar la variable de campo académico con el propósito de indagar sobre los patrones ecológicos vinculados al estudio de una profesión.

Además de concientizar a los estudiantes para adoptar personalmente una cultura ecocéntrica, se espera que los estudiantes adquieran conocimientos del mundo natural incluyendo flora, fauna y la observación científica de la misma desarrollando la inteligencia ecológica.

Para finalizar, se espera estudiar a la muestra seleccionada a través de la Escala de Actitudes y Preocupación Ambiental. La escala se compone de 30 ítems que aportan información acerca de las dimensiones de Egoísmo, Socioaltruismo, y Ego-biocentrismo (Palavecinos, Amérigo, \& Muñoz, 2010, 4), con el objetivo de evaluar con mayor cabalidad qué visión ambiental predomina en los sujetos de estudio para conseguir resultados más puntuales que los obtenidos en él.

\section{Bibliografia}

Acevedo, F. (2010). Cultura y nociones conexas: Algunas consideraciones antropológicas. Recuperado el 5 de abril de 2015, de http://www.dfpd.edu.uy/departamentos/sociologia/adjuntos/cultura.pdf
Asamblea General de las Naciones Unidas. (1987). Informe Brundtland. Recuperado el 22 de abril de 2015, de Desarrollo Sostenible: http://www.un.org/es/ga/ president/65/issues/sustdev.shtml

Astudillo, E. (2010). El desarrollo sostenible comunitario en un ENP con alto interés científico: "El caso de la Comuna Loma Alta y su Reserva Ecológica" (Tesis de Maestría no publicada). Madrid: Universidad Autónoma de Madrid.

Cordera, R. (2009). La Universidad Latinoamericana y la Crisis Global. México: Universidades de América Latina y el Caribe.

Corral-Verdugo, V., \& Pinheiro, J. (2004). Aproximaciones al estudio de la conducta sustentable: Medio Ambiente y Comportamiento Humano. México: Revista Mexicana de Investigación Educativa.

De la Torre, L. N. (2008). Enciclopedia de las plantas útiles del Ecuador: Quito: Herbario QCA de la Escuela de Ciencias Biológicas de la Pontificia Universidad Católica del Ecuador \& Herbario AAU del Departamento de Ciencias Biológicas de la Universidad de Aarhus.

Douglas, N. (2009). Reading Explorer: Urban Challenges. Canadá: Heinle Cengage Learning.

ECOLAP \& MAE. (2007). Guia del Patrimonio de Areas Naturales Protegidas del Ecuador. Recuperado el 5 de abril de 2015, de http://blog.espol.edu.ec/ricardomedina/files/2009/03/37-portada20general20ultimas20pags.pdf

Edinun. (2009). Ecuador Ecológico: Texto de Educación Ambiental. Quito: Ediciones Nacionales Unidas.

El Tiempo. (20 de marzo de 2015). Madre Tierra promueve la cultura ecológica. $E l$ tiempo, págs. http://www.eltiempo.com.ed noticias-cuenca/159257-madre-tierra-promueve-la-cultura-ecola-gical. 
Escalera, J., \& Ruiz, E. (2011). Resiliencia Socioecológica: Aportaciones y Retos desde la Antropología. Sevilla: Universidad Pablo de Olavide.

FAO. (2002). El estado mundial de la pesca y acuicultura. Recuperado el 22 de abril de 2015, de Departamento de Pesca: ftp://ftp.fao.org/docrep/fao/005/y $7300 s / y 7300 s . p d f$

Goleman, D. (2009). Inteligencia Ecológica. Buenos Aires: Vergara.

Grotberg. (2006). La resiliencia en el mundo de hoy: Cómo superar las adversidades. Barcelona: Gedisa Editorial.

Grupo Edebé. (2008). Filosofia y Ciudadanía. Barcelona: Ormograf.

Harper \& Row. Inc. (2009). Antropología Cultural. Madrid: Alianza Editorial.

Hernández, M. (2009). La resiliencia de los ecosistemas, clave del desarrollo sostenible. Recuperado el 30 de noviembre de 2014, de CEGESTI: http://www.cegesti.org/exitoempresarial/publicaciones/publicacion_99_301109_es.pdf

Hernández, R., Fernández, C., \& Baptista, M. (2010). Metodología de la Investigación. México: Mc Graw Hill.

Leff, E. (2009). Racionalidad Ambiental: La reapropiación social de la naturaleza. México: Siglo XXI Editores S.A.

Maaluf, A. (2009). Identidades Asesinas. (F. Villaverde, Ed.) Madrid: Alianza Editorial.

Marchisio, A., Devesa, H., Rosso, C., \& Sica, F. (2012). La Evolución Biológica: Actualidad y Debates. Buenos Aires: Ministerio de Educación de la Nación.

Miller, G. J. (2007). Ciencia ambiental : desarollo sostenible, un enfoque integral. México: Cengage Learning Latino América.
Mosterín, J. (2008). Lo mejor posible: Racionalidad y Acción Humana. Madrid: Alianza Editorial.

Myers, D. (2011). Psicología. España: Editorial Médica Panamericana.

Novo, M. (2006). El desarrollo sostenible: Su dimensión ambiental y educativa. Ma-drid: Pearson Educación S.A. Madrid : Pearson Educación S.A.

Nuñez, J. (2012). Psicología: Acerca de ti . Madrid: Ediciones Paraninfo.

Palavecinos, M., Amérigo, M., \& Muñoz, J. (2010). Preocupación y Conducta Ecológica Responsable en estudiantes Universitarios: El rol de la Universidad en la Educación Ambiental. Chile: Universidad de la Friontera.

Papalia, D., Wendkos, S., \& Duskin, R. (2010). Desarrollo Humano. México D.F: McGraw Hill Educación Ediciones Latinoamericanas S.A.

Programa de las Naciones Unidas para el Medio Ambiente. (2007). Perspectivas del Medio Ambiente Mundial: GEO 4 - Medio Ambiente para el desarrollo. Dinamarca: Phoenix Design Aid.

Reyes, G., \& Martí, S. (septiembre de 2007). revistaecosistemas.net. Recuperado el 23 de enero de 2015, de Asociación Española de Ecología Terrestre: http://ocw.um. es/ciencias/avances-ecologicos-para-lasostenibilidad-de-los/lectura-obligatoria-1/leccion 11/etnoecologia.pdf

Salazar, F. (1991). El concepto de cultura y los cambios culturales. Sociológica, VI, 17.

Sánchez, A. (2011). Conceptos básicos de Gestión Ambiental y Desarrollo Sustentable. México: Instituto Nacional de Ecología.

Simental, V. (2010). Derecho Ambiental. México: Limusa S.A. 
Terradas, J. (2001). Ecología Urbana. Barcelona: Rubes Editorial.

UNESCO. (1982). Conferencia Mundial sobre las Políticas Culturales. DECLARACIÓN DE MÉXICO SOBRE LAS POLÍTICAS CULTURALES. México.

Valls, M. (2008). Derecho Ambiental. Buenos Aires: Abeledo Perrot.

Vitarelli, M. F. (2007). Formación docente e investigación: Propuestas en Desarrollo. Argentina: Universidad de San Luis.

World Wide Fund. (2014). Infome Planeta Vivo: Resumen. Cali, Colombia.

\section{Anexos}

Modelo de encuesta a estudiantes:

\section{Encuesta Dirigida a Estudiantes de la UEES \\ Estimados Compañeros:}

Los estudiantes de la materia de Ecología, estamos elaborando un proyecto aúlico con el tema "Cultura Ecológica en Jóvenes Universitarios". Sus opiniones servirán para analizar e identificar el desarrollo de la conciencia ecológica y la motivación por la investigación científica en la institución. Indicamos, además, que esta encuesta es confidencial y anónima; cuyos resultados serán conocidos de manera tabulada e impersonal.

Edad:

Carrera que cursa:

Género:

$€$ Femenino

$€ \quad$ Masculino

1. ¿Es el medio ambiente importante para ti?

Marca sólo una opción de respuesta

$€ \quad$ Definitivamente sí
$€ \quad$ Probablemente sí
$€$ Indeciso
$€$ Probablemente no
$€$ Definitivamente no

2. Ordene las siguientes opciones con sus respectivos números según la influencia que has recibido acerca del cuidado del medio ambiente

1.Familia , 2.Amigos/Conocidos, 3.Tus profesores, 4.Tu facultad, 5.Opiniones de Expertos Responda en el siguiente recuadro:

3. ¿De qué manera han influido en tu percepción para el cuidado del medio ambiente?

Responde en relación a tu respuesta anterior

$€$ Con actividades de reciclaje, reuso y reutilizar

$€$ Foros - debates sobre temas ambientales

$€$ Valoración a la naturaleza (Ejm: Cuidar el jardín, actividades al aire libre, etc)

$€$ Otros ¿Cuál?

4. ¿Consideras que tus actividades diarias contaminan al entorno en que te desenvuelves?

Marca sólo una opción de respuesta
$€$ Definitivamente sí
$€ \quad$ Probablemente sí
$€$ Indeciso
$€$ Probablemente no
$€$ Definitivamente no

5. ¿Qué actividades para cuidar el medio ambiente realizas?

Escoge al menos 3 opciones (excepto si escoges "Ninguna") y ordénalas en orden de importancia

1.Venta de Reciclaje de periódicos, 2.Uso de focos 
aborradores en el hogar, 3.Medidas para ahorrar el agua, 4. Reciclaje y/o Venta de botellas de plástico/vidrio, 5.Uso de gasolina ecológica, 6.Elaboración de manualidades con materiales reciclados, 7.No depositar basura en las calles, 8.Ninguna

Responda en el siguiente recuadro:

6. ¿Crees que los jóvenes de hoy deberíamos tomar acción en actividades ecológicas?

Marca sólo una opción de respuesta

$€$ Definitivamente sí

$€ \quad$ Probablemente sí

$€$ Indeciso

$€ \quad$ Probablemente no

$€$ Definitivamente no

7. De las siguientes opciones elije a tu criterio, cuál se ajusta al término de desarrollo sustentable.

$€ \quad$ Es el consumo excesivo e irracional de los recursos naturales.

$€ \quad$ Es la preservación de los recursos naturales a corto plazo para beneficio exclusivo del hombre.

$€ \quad$ Comprende el equilibrio entre el modo de vida humana y la preser vación de los recursos naturales para las generaciones futuras.

\section{Evelyng Astudillo Sainchez}

Bióloga y Máster en Espacios Naturales Protegidos. Docente tiempo completo de la Universidad Espiritu Santo - UEES

E-mail: eastudillo@uees.edu.ec

\section{Nicole Melendres Vélez}

Estudiante de Psicología Clínica de la Universidad Espiritu Santo - Ecuador

E-mail: nikymelendres@uees.edu.ec

\section{Brunny Espinoza Amén}

Estudiante de Ciencias Ambientales de la Universidad Espiritu Santo - Ecuador

E-mail: bespinoza@uees.edu.ec

\section{Marco Moscoso Chávez}

Estudiante de Psicologia Clinica de

la Universidad Espiritu Santo - Ecuador

E-mail: andiemoscoso@ uees.edu.ec

\section{Teresa Baquerizo Cornejo}

Psicóloga Clinica y Máster en Terapia Familiar con mención en Psicoterapia Sistemática Docente tiempo completo de la Universidad Espiritu Santo - UEES

E-mail: tbaquerizo@uees.edu.ec 
